\title{
Production of interleukin-1 like activity by neutrophils derived from rat lung
}

\author{
Yukinori Kusaka, Kenneth Donaldson
}

\begin{abstract}
Interleukin-1 like activity was produced -by neutrophils obtained by bronchoalveolar lavage from experimentally inflamed rat lung. Activity was released spontaneously from neutrophils at high levels but it was enhanced by stimulation with endotoxin in vitro.
\end{abstract}

The role of the alveolar macrophage in immune and inflammatory responses in the lung has been well documented. ${ }^{1}$ During inflammatory responses in the lung, however, other leucocyte types are recruited to the alveolar region, including polymorphonuclear neutrophils. ${ }^{2}$ changes that follow inflammation in the lung in the adult respiratory distress syndrome, idiopathic pulmonary fibrosis, and lung disease related to mineral dusts. Some inflammatory lung diseases, including idiopathic pulmonary fibrosis and extrinsic allergic alveolitis, have an important immunological component, whereas diseases such as pneumoconiosis have a less obvious immunological element. In all these diseases, however, neutrophils are found in increased numbers in bronchoalveolar lavage fluid. Hitherto the neutrophil has not been considered to have a major influence on the immune responses in the lung, but we report neutrophil derived interleukin-1 like activity in cells obtained from inflamed rat lungs.

\section{Methods \\ RATS \\ We used 12-15 week old female specific pathogen free, inbred PVG rats from the Institute of Occupational Medicine's own breeding unit.}

INDUCTION OF INFLAMMATION IN THE LUNG Pulmonary inflammation was induced by transtracheal instillation of $1 \mathrm{mg}$ of quartz $\left(\mathrm{DQ}_{12}\right.$ standard) or $1.4 \mathrm{mg}$ of a heat killed preparation of Corynebacterium parvum (Wellcome, Beckenham). One to seven days later the lungs were lavaged and bronchoalveolar cells obtained. ${ }^{3}$ The lungs of control rats were lavaged to obtain normal bronchoalveolar cells ( $>95 \%$ alveolar macrophages).

SEPARATION OF CELLS

Whole inflammatory bronchoalveolar cell preparations were separated into neutrophil rich populations by centrifugation through Sepracell medium (Sepracell, Oklahoma). These have been implicated in the pathological

MEASUREMENT OF INTERLEUKIN-1

Interleukin-1 like activity was determined in dilutions of supernatant from overnight cultures of the whole or separated cell populations in the presence or absence of lipopolysaccharide (Escherichia coli, serotype 0111: B4, 100 ng/ml; Sigma, Poole). Cells were cultured at $37^{\circ} \mathrm{C}$ in RPMI 1640 with $10 \%$ fetal calf serum (Gibco, Paisley). Dilutions of supernatant were incubated with $\mathrm{C} 3 \mathrm{H}$ mouse thymocytes at $6 \times 10^{5}$ cells $/$ well in microtitre plates. Phytohaemagglutinin was added to a final concentration of $4 \mu \mathrm{g} / \mathrm{ml}$, a concentration previously determined to be suboptimal, and the plates were cultured for 72 hours. Thymocyte proliferation was determined by the incorporation of tritiated thymidine added during the final 16 hours of culture. Controls included wells without supernatant and wells with a supernatant collected from C57B16 mouse peritoneal macrophages cultured with $10 \mu \mathrm{g} / \mathrm{ml}$ lipopolysaccharide recombinant interleukin-1 $\alpha$.

\section{STATISTICAL ANALYSIS}

Differences between treatment groups were analysed by Student's $t$ test.

\section{Results}

INTERLEUKIN-1 LIKE ACTIVITY

In all experiments the background level of thymocyte proliferation produced counts of $500-1000 \mathrm{cpm}$ and the two positive controls counts ranging from 3500 to $12500 \mathrm{cpm}$. The neutrophil rich populations obtained from rats treated with $C$ parvum and from rats instilled with quartz produced substantial quantities of interleukin-1 like activity; in both cases the proportion of neutrophil approached $80 \%$ (fig 1). Interleukin-1 like activity was also produced by alveolar macrophages. Although substantial, this could not account for the increased interleukin-1 like activity produced by the neutrophil enriched populations (fig 1). In one experiment the neutrophils from rats treated with $C$ parvum were enriched in Sepracell separation medium, which increased their proportion from $76 \%$ to $100 \%$. This caused the mean interleukin-1 like activity to increase from 2094 (SD 107) to 2604 (198) cpm.

Incubation of control macrophages or an

Address for reprint requests: Dr Kenneth Donaldson, Institute of Occupational Medicine, Edinburgh EH8 9SU.

Accepted 23 November 1989
$83 \%$ pure neutrophil population with lipopolysaccharide (100 $\mathrm{ng} / \mathrm{ml})$ produced a substantial stimulation of interleukin-1 like activity (fig 2 ). 


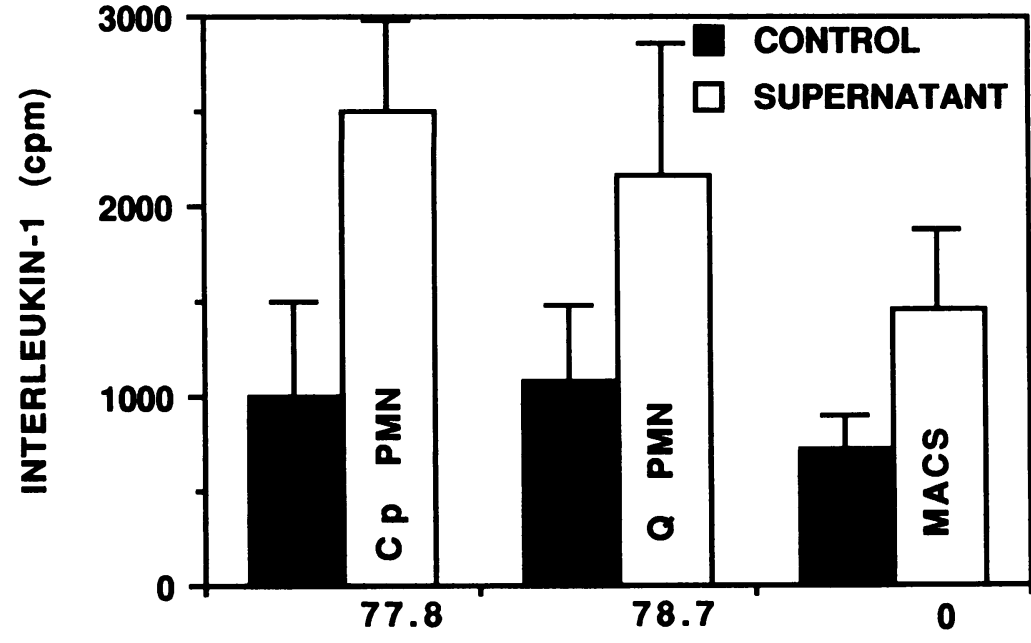

Figure 1 Presence of interleukin-1 like activity in culture supernatants of polymorphonuclear neutrophils obtained from Corynebacterium parvum stimulated lung (Cp PMN) and quartz stimulated lung (Q PMN); supernatants from alveolar macrophages (MACS) are included for comparison. Significant $(p<0.001)$ increases occurred with leucocyte populations compared with thymocyte controls (black columns) for each sequence of experiments. Data are derived from triplicate wells in fout (Cp PMN), six (Q PMN) or three (MACS) separate experiments. Numbers underneath the open bars indicate the percentage of neutrophils in the culture.

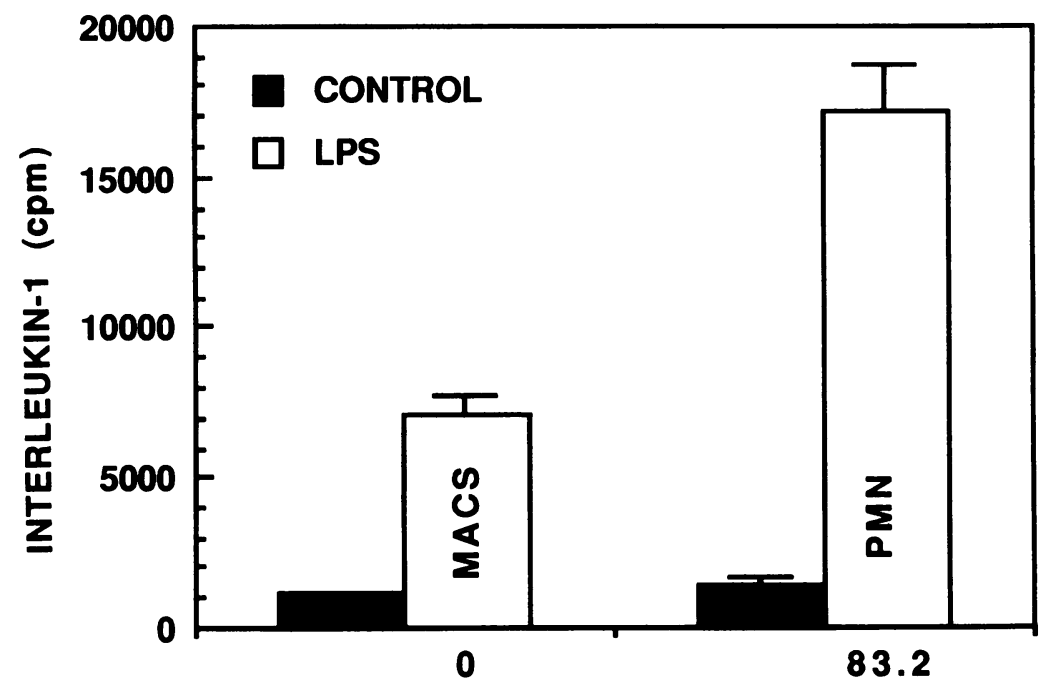

Figure 2 Presence of interleukin-1 like activity in supernatants obtained from alveolar macrophages (MACS) and Corynebacterium parvum (PMN), cells being unstimulated or stimulated by incubation with lipopolysaccharide (LPS) $(100 \mathrm{ng} / \mathrm{ml})$ for 24 hours.

There were significant increases $(p<0.001)$ from treatment with lipopolysaccharide. Means and SEM of results from triplicate wells are given. Numbers under the open bars indicate the percentage of neutrophils.

EXCLUSION OF THE CONTRIBUTION OF OTHER CYTOKINES IN THE THYMOCYTE ASSAY

As other cytokines could be present in the supernatants and could enhance thymocyte responses we sought to examine whether interleukin-2 or tumour necrosis factor was present. Using the interleukin-2 sensitive cell line CTTL-2, we found no interleukin-2 activity in any supernatant. Recombinant tumour necrosis factor had negligible activity in the thymocyte assay.

\section{Discussion}

Interleukin-1 derived from neutrophils is potentially important in the alveolitis caused by quartz (silica). ${ }^{4}$ We believe that neutrophil derived cytokines could be important in inflammatory lung disease and so report the production of interleukin-1 like activity by neutrophils from rat lung inflamed by quartz and heat killed bacteria.

Neutrophils derived from the inflamed lung are capable of producing large amounts of interleukin-1 like activity as assessed in the thymocyte enhancement assay. We excluded interleukin-2 and tumour necrosis factor as likely contaminating activities in the thymocyte assay. Neutrophils are found in the alveolar region and in the alveolar spaces in a range of diseases in which an immunological component is suspected. The release of interleukin1 like activity by neutrophils could modulate the function of lymphocytes and other cells in the lung parenchyma and in the lung lymph nodes during inflammation and potentially cause local and systemic modulation of the immune response.

Neutrophils occur in the lungs of humans and animals exposed to non-antigenic mineral dusts such as asbestos and quartz. ${ }^{4}$ They might be important in the local and systemic immune changes reported in dust exposed workers and experimental animals, which could be important in the disease process. Interleukin-1 is a fibroblast growth factor as well as having multiple effects on other cells, including endothelial cells and leucocytes. ${ }^{5}$ If the interleukin-1 like activity is derived from neutrophils, this has important potential consequences for understanding pathological changes occurring in a range of inflammatory lung diseases.

Interleukin- 1 can be produced and released by neutrophils from the rabbit peritoneal cavity $^{6}$ and from both bovine ${ }^{7}$ and human ${ }^{8}$ peripheral blood. This is to our knowledge the first report of interleukin-1 like activity in neutrophils derived from experimentally inflamed lung.

This work was funded by the Commission of the European Communities and the Colt Foundation, and YK had a personal grant from the Colt Foundation.

\footnotetext{
1 Hunninghake GW, Gadek JE, Kawanami O, Ferrans J, Crystal RG. Inflammatory and immune processes in the human lung in health and disease. Evaluation by bronchoalveolar lavage. Am J Pathol 1979;97:149-206.

2 Keogh BA, Crystal RG. Alveolitis-the key to the interstitial lung disorders. Thorax 1982;37:1-10.

3 Donaldson $\mathrm{K}$, Bolton RE, Jones $\mathrm{AD}$, et al. Kinetics of the bronchoalveolar leukocyte response in rats following exposure to equal airborne mass concentrations of quartz, chrysotile asbestos or titanium dioxide. Thorax 1988 43:525-33.

4 Kusaka Y, Cullen RT, Donaldson K. Immunomodulation in mineral dust-exposed lung: stimulatory effect and interleukin-1 release by neutrophils from quartz-elicited alveolitis. Clin Exp Immunol (in press).

5 artin M. Resch K. Interleukin-1-more than a mediator between leukocytes. Trends Pharmacol Sci 1988;9:171-7.

6 Goto F, Goto K, Ohkawara S, et al. Purification and partial sequence of rabbit polymorphonuclear leukocyte-derived lymphocyte proliferation potentiating factor resemblin IL-1. J Immunol 1988;140:1153-8.

7 Canning PC, Neill JD. Isolation and characterisation of interleukin-1 from bovine polymorphonuclear leukocytes. J Leukocyte Biol 1989;45:21-8.

8 Tiku K, Tiku ML, Skosey JL. Interleukin-1 production by human polymorphonuclear neutrophils. $J$ Immunol 1986;
} 136:3677-80. 